\title{
Risk for metabolic syndrome in the population with visceral fat area measured by bioelectrical impedance analysis
}

\author{
Han Ho Jeon ${ }^{1,}$, Yong Kang Lee ${ }^{1,}$, Dong Hyun Kim ${ }^{1}$, Haeyong Pak ${ }^{2}$, Sang Yun Shin ${ }^{1}$, and Jeong Hun Seo ${ }^{1}$
}

\begin{abstract}
${ }^{1}$ Department of Internal Medicine, ${ }^{2}$ Institute of Health Insurance and Clinical Research, National Health Insurance Service Ilsan Hospital, Goyang, Korea
\end{abstract}

Received: November 28, 2018 Revised : May 24, 2019

Accepted: June 2, 2019

\section{Correspondence to} Jeong Hun Seo, M.D.

Department of Internal Medicine, National Health

Insurance Service Ilsan Hospital, 100 Ilsan-ro, Ilsandong-gu,

Goyang 10444, Korea

Tel: +82-031-900-0974

Fax: +82-031-900-6967

E-mail:jhsuh@nhimc.or.kr

*These authors contributed equally to this work.
Background/Aims: To investigate whether visceral fat area (VFA) measured by bioelectric impedance analysis (BIA) was associated with metabolic syndrome in subjects with and without obesity.

Methods: A total 23,202 participants who underwent medical check-ups were assessed. Participants were stratified by body mass index (BMI) and VFA. We evaluated six different groups for metabolic syndrome: Group 1 (normal weight and low VFA), Group 2 (normal weight and high VFA), Group 3 (overweight and low VFA), Group 4 (overweight and high VFA), Group 5 (obesity and low VFA), and Group 6 (obesity and high VFA).

Results: Metabolic syndrome traits and metabolic syndrome were significantly more prevalent in the high-VFA $\left(\geq 100 \mathrm{~cm}^{2}\right)$ subgroup in each BMI group. Adjusted logistic regression analyses revealed that the odds ratio for metabolic syndrome compared with Group 1 was the highest in Group 6 (24.53; 95\% confidence interval [CI], 21.77 to 27.64). Notably, the odds ratio of Group 2 was higher than that of Group 3 (2.92; 95\% CI, 2.30 to 3.69 vs. 2.57 ; $95 \%$ CI, 2.23 to 2.97 ).

Conclusions: Our study demonstrates that the combination of BMI assessment and VFA determination by BIA may be a useful method for predicting the risk of metabolic syndrome. The VFA by BIA may be a useful target for interventions to improve metabolic syndrome.

Keywords: Intra-abdominal fat; Metabolic syndrome; Electric impedance

\section{INTRODUCTION}

Due to recent changes in eating habits and lifestyle, the prevalence of obesity is increasing worldwide $[1,2]$, regardless of race, age, and gender, and the incidence rates and complications of obesity-related diseases are increasing. People with obesity are more likely to develop metabolic disorders such as metabolic syndrome, type 2 diabetes, hypertension, dyslipidemia, ischemic heart disease, stroke, fatty liver, gallbladder disease, and thyroid disease [2-4].
Visceral fat plays an important role in the development of metabolic and cardiovascular diseases [5-7]. However, body mass index (BMI), the main measure used to determine obesity, does not account for the amount of muscle and fat, but simply accounts for body weight [8]. Therefore, there is a limit to predicting obesity-related disease. In addition, it is possible to evaluate abdominal obesity through the measurement of waist circumference [9], but there are limitations in that body fat percentage cannot be measured directly, and the reproducibility is poor due to large errors in the measurements 
[10]. Methods for measuring visceral fat area (VFA) include dual energy X-ray absorptiometry, computed tomography (CT), and magnetic resonance imaging; however, these methods require expensive equipment and exposure to radiation, which limits their use. Current body composition analysis through bioelectrical impedance analysis (BIA) is the most widely used method because it has the advantage of measuring body fat and muscle mass easily and inexpensively [11-13].

In this study, we analyzed the relationship between metabolic syndrome in the obesity group defined by BMI and the obesity group defined by the VFA determined by bioelectrical impedance and assessed the risk of metabolic syndrome by subdividing the groups according to the combination of BMI and VFA.

\section{METHODS}

\section{Study population}

We identified 43,837 adults ( $\geq 18$ years old) who underwent voluntary routine check-ups at the National Health Insurance Service Ilsan Hospital between January 2011 and December 2015. When participants underwent multiple examinations, we analyzed the data from their first visit. We excluded participants with BMI less than 18.5 $\mathrm{kg} / \mathrm{m}^{2}$. In total, 23,202 participants were included in this study. This study was approved by the Institutional Review Board of the National Health Insurance Service Ilsan Hospital (IRB No.: 2017-04-043-002). Written informed consent by the patients was waived due to a retrospective nature of our study.

\section{Data collection}

The participants arrived at the hospital after an overnight fast. Clinical and laboratory data were collected during the health examination. The height and weight of each participant were measured while he/she maintained a straight standing posture while wearing a light examination suit and no shoes, and the BMI was calculated. The waist circumference was measured mid-way between the lowest rib and the iliac crest. Visceral fat area was measured by a trained nurse using an InBody 720 (Biospace Co., Seoul, Korea) according to the manufacturer's protocol. The following clinical and laboratory data were collected: age, body weight $(\mathrm{kg})$, body mass index, smoking, drinking habits, waist circumference (cm), systolic blood pressure $(\mathrm{mmHg})$, diastolic blood pressure $(\mathrm{mmHg})$, uric acid $(\mathrm{mg} / \mathrm{dL})$, total cholesterol (mg/dL), high-density lipoprotein cholesterol (HDL-C; $\mathrm{mg} / \mathrm{dL}$ ), low-density lipoprotein cholesterol (LDL-C; $\mathrm{mg} / \mathrm{dL})$, triglycerides $(\mathrm{mg} / \mathrm{dL})$, fasting glucose $(\mathrm{mg} / \mathrm{dL})$, hemoglobin Aic (HbAic, \%), gamma-glutamyl transferase (GGT, U/L), aspartate aminotransferase (AST, U/L), and alanine aminotransferase (ALT, U/L).

\section{Definitions}

The cut-off point for obesity for Asians is defined by the World Health Organization as a BMI of $23.0 \mathrm{~kg} / \mathrm{m}^{2}$ [14]. The participants were divided into three groups by BMI: normal weight (18.5 to $22.9 \mathrm{~kg} / \mathrm{m}^{2}$ ), overweight (23 to 24.9 $\left.\mathrm{kg} / \mathrm{m}^{2}\right)$, and obesity $\left(\geq 25 \mathrm{~kg} / \mathrm{m}^{2}\right)$. The definition of obesity by VFA was set as VFA $\geq 100 \mathrm{~cm}^{2}[15]$.

Participants were categorized into six groups according to the combination of BMI and VFA. The six groups were (Fig. 1): (1) normal weight and VFA $<100 \mathrm{~cm}^{2}$ (Group 1); (2) normal weight and VFA $\geq 100 \mathrm{~cm}^{2}$ (Group 2); (3) overweight and VFA $<100 \mathrm{~cm}^{2}$ (Group 3); (4) overweight and VFA $\geq 100 \mathrm{~cm}^{2}$ (Group 4); (5) obesity and VFA $<100 \mathrm{~cm}^{2}$ (Group 5); and (6) obesity and VFA $\geq 100 \mathrm{~cm}^{2}$ (Group 6).

Metabolic syndrome was defined in accordance with the National Cholesterol Education Program Expert Panel on Detection, Evaluation, and Treatment of High Blood Cholesterol in Adults, Adult Treatment Panel III. Participants were defined as having metabolic syndrome if they had three or more of the following five factors: (1) waist circumference: male $\geq 90 \mathrm{~cm}$, female $\geq 80 \mathrm{~cm}$; (2) triglycerides: $\geq 150 \mathrm{mg} / \mathrm{dL}$ or use of medication; (3) HDL-C: male $<40 \mathrm{mg} / \mathrm{dL}$, female $<50 \mathrm{mg} / \mathrm{dL}$ or use of medication; (4) blood pressure $\geq 130 / 85 \mathrm{mmHg}$ (systolic blood pressure $\geq 130 \mathrm{mmHg}$ or diastolic blood pressure $\geq 85 \mathrm{mmHg}$ ) or use of medication; and (5) fasting glucose $\geq 100 \mathrm{mg} / \mathrm{dL}$ or use of medication.

\section{Statistical analysis}

Continuous variables are presented as mean \pm standard deviation. Categorical variables are presented as count (percentage). Differences between groups were tested by Student's $t$ test for continuous variables and the chisquare test for categorical variables. Multiple logistic regression analysis was used to determine the risk of metabolic syndrome in each of the six groups. First, we used 


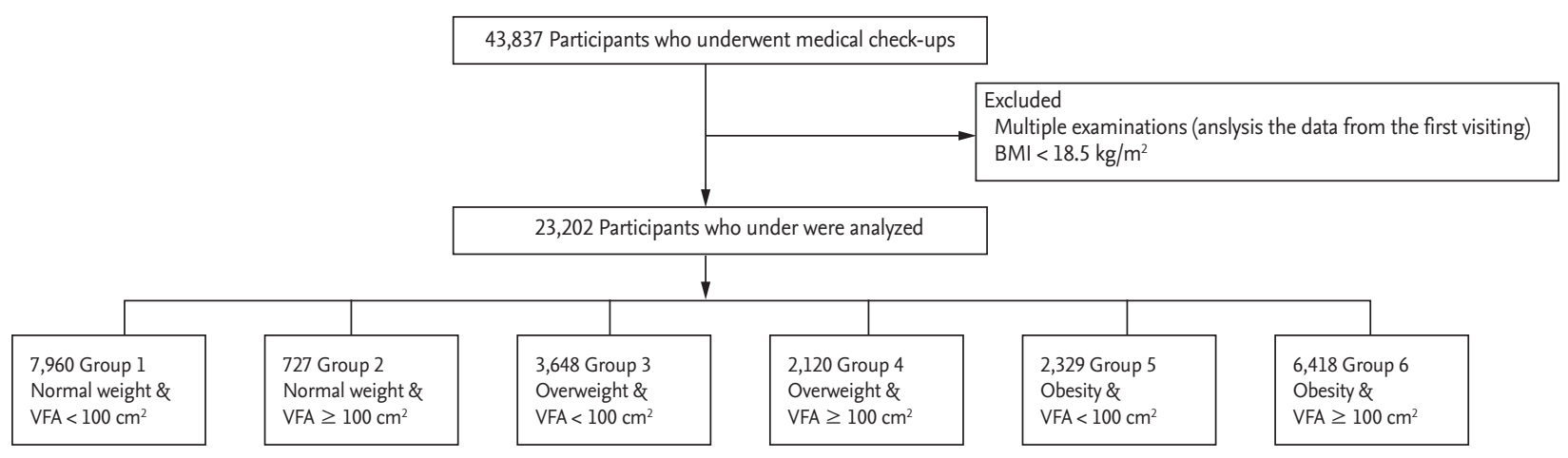

Figure 1. Study participant and categorization. Normal weight, body mass index (BMI) 18.5 to $22.9 \mathrm{~kg} / \mathrm{m}^{2}$; overweight, BMI 23 to $24.9 \mathrm{~kg} / \mathrm{m}^{2}$; obesity, BMI $\geq 25 \mathrm{~kg} / \mathrm{m}^{2}$. VFA, visceral fat area.

Table 1. Clinical characteristics of the study population stratified by gender

\begin{tabular}{|c|c|c|c|}
\hline Characteristic & Male $(\mathrm{n}=12,772)$ & Female $(n=10,430)$ & $p$ value \\
\hline Age, yr & $47 \cdot 9 \pm 11.9$ & $47.6 \pm 12.5$ & 0.028 \\
\hline Body weight, kg & $73.5 \pm 10.7$ & $58.4 \pm 8.3$ & $<0.001$ \\
\hline BMI, $\mathrm{kg} / \mathrm{m}^{2}$ & $25.0 \pm 3.0$ & $23.3 \pm 3.2$ & $<0.001$ \\
\hline Waist circumference, $\mathrm{cm}$ & $86.6 \pm 7.8$ & $80.6 \pm 8.2$ & $<0.001$ \\
\hline $\mathrm{VFA}, \mathrm{cm}^{2}$ & $106.5 \pm 35.6$ & $82.2 \pm 31.3$ & $<0.001$ \\
\hline Hypertension & $14,02(11.0)$ & $674(6.5)$ & $<0.001$ \\
\hline Diabetes mellitus & $2,229(17.5)$ & $1,111(10.7)$ & $<0.001$ \\
\hline Smoking $^{\mathrm{a}}$ & $9,666(75 \cdot 7)$ & $745(7.1)$ & $<0.001$ \\
\hline Alcohol drinking ${ }^{\mathrm{b}}$ & $4,842(37 \cdot 9)$ & $3,298(31.6)$ & $<0.001$ \\
\hline Systolic BP, mmHg & $123.7 \pm 13.9$ & $117 \cdot 39 \pm 15 \cdot 1$ & $<0.001$ \\
\hline Diastolic BP, mmHg & $75 \cdot 9 \pm 10.6$ & $71.49 \pm 10.5$ & $<0.001$ \\
\hline Total cholesterol, mg/dL & $198.7 \pm 37.5$ & $195.8 \pm 36.3$ & $<0.001$ \\
\hline $\mathrm{HDL}-\mathrm{C}, \mathrm{mg} / \mathrm{dL}$ & $47 \cdot 9 \pm 11.4$ & $56.67 \pm 13.4$ & $<0.001$ \\
\hline LDL-C, mg/dL & $121.6 \pm 33.2$ & $116.85 \pm 32.3$ & $<0.001$ \\
\hline Triglyceride, mg/dL & $153.2 \pm 112.7$ & $98.23 \pm 65.5$ & $<0.001$ \\
\hline $\mathrm{HbA1c}, \%$ & $5.8 \pm 0.8$ & $5.7 \pm 0.7$ & $<0.001$ \\
\hline Metabolic syndrome & $3,799(29 \cdot 7)$ & $2,046(19.6)$ & $<0.001$ \\
\hline Fasting blood glucose, mg/dL & $99.1 \pm 23.5$ & $94.1 \pm 17.5$ & $<0.001$ \\
\hline Uric acid, mg/dL & $6.0 \pm 1.2$ & $4.4 \pm 0.9$ & $<0.001$ \\
\hline GGT, IU/L & $39.8 \pm 64.2$ & $17.5 \pm 15.7$ & $<0.001$ \\
\hline AST, IU/L & $27.5 \pm 14.5$ & $22.7 \pm 9.9$ & $<0.001$ \\
\hline ALT, IU/L & $30.8 \pm 23.5$ & $20.0 \pm 13.4$ & $<0.001$ \\
\hline
\end{tabular}

Values are presented as mean \pm SD or number (\%).

BMI, body mass index; VFA, visceral fat area; BP, blood pressure; HDL-C, high-density lipoprotein cholesterol; LDL-C, low-density lipoprotein cholesterol; HbAıc, hemoglobin Aıc; GGT, gamma-glutamyl transferase; AST, aspartate transaminase; ALT, alanine transaminase.

${ }^{a}$ Smoking: past smoker and current smoker.

${ }^{\mathrm{b}}$ Alcohol drinking: drink more than once a week. 
Table 2. Correlation between VFA and other variables

\begin{tabular}{lc}
\hline Variable & Correlation coefficient \\
\hline Age & 0.209 \\
BMI & 0.701 \\
Waist circumference & 0.793 \\
Systolic BP & 0.349 \\
Diastolic BP & 0.307 \\
Total cholesterol & 0.148 \\
HDL-C & -0.303 \\
LDL-C & 0.174 \\
Triglyceride & 0.322 \\
Fasting glucose & 0.229 \\
HbAic & 0.228 \\
Uric acid & 0.340 \\
GGT & 0.166 \\
AST & 0.223 \\
ALT & 0.303 \\
\hline
\end{tabular}

Values are the correlation coefficients and $p<0.001$ for all analyses.

VFA, visceral fat area; BMI, body mass index; BP, blood pressure; HDL-C, high-density lipoprotein cholesterol; LDL-C, low-density lipoprotein cholesterol; HbA1c, hemoglobin Aıc; GGT, gamma-glutamyl transferase; AST, aspartate transaminase; ALT, alanine transaminase.

the base model for the BMI and VFA at baseline. Model 1 was adjusted for age and sex, while Model 2 was adjusted for age, sex, diabetes mellitus, hypertension, alcohol intake, and smoking. The associations are presented as odds ratios (ORs) with 95\% confidence intervals (CIs). All statistical analyses were performed with SAS 9.4 (SAS Institute, Cary, NC, USA). A p value below 0.05 was considered statistically significant.

\section{RESULTS}

\section{Baseline characteristics of study population}

The demographic and clinical characteristics of the participants are shown in Table 1. The mean age was 47.6 years for women and 47.9 years for men. The mean VFA was $82.2 \mathrm{~cm}^{2}$ in women and $106.5 \mathrm{~cm}^{2}$ in men. The prevalence of metabolic syndrome in women and men was $19.6 \%$ and $29.7 \%$ respectively. The mean BMI, blood pressure, uric acid, total cholesterol, LDL-C, triglycerides, fasting blood glucose, GGT, AST, and ALT levels were significantly higher in men than in women. The mean HDL-C level was significantly higher in women than in men. Table 2 displays the correlations between VFA and various other parameters. The VFA correlated positively with BMI, blood pressure, uric acid, total cholesterol, LDL-C, triglycerides, fasting blood glucose, GGT, AST, and ALT levels, but negatively with HDL-C level.

\section{Main characteristics of the groups defined by BMI and VFA}

We divided participants into six groups according to the combination of BMI and VFA. The clinical characteristics of participants by gender according to BMI and VFA are shown in Tables 3 and 4. In men, the prevalence of metabolic syndrome was $6.1 \%$ in Group 1, $13.3 \%$ in Group 2, 13.1\% in Group 3, 21.5\% in Group 4, 25.4\% in Group 5, and 56.2\% in Group 6. In women, the prevalence of metabolic syndrome was $4.3 \%$ in Group 1, 21.6\% in Group 2, $12.1 \%$ in Group 3, 30.9\% in Group 4, 33.9\% in Group 5 and $62.2 \%$ in Group 6. The subgroups of participants with VFA $\geq 100 \mathrm{~cm}^{2}$ were generally older and had greater BMI and waist circumference than those with VFA $<100 \mathrm{~cm}^{2}$. In addition, VFA $\geq 100 \mathrm{~cm}^{2}$ was associated with the presence of hypertension, diabetes mellitus, metabolic syndrome, and metabolic parameters.

\section{Presence of metabolic syndrome in groups defined by BMI and VFA}

In variable-adjusted models, we estimated the risk of metabolic syndrome in each group defined by BMI and VFA (Table 5). In Model 1, the odds ratios for metabolic syndrome compared with Group 1 were 2.91 (95\% CI, 2.31 to 3.67 ), 2.64 (95\% CI, 2.29 to 3.04), 5.27 (95\% CI, 4.55 to 6.11), 7.62 (95\% CI, 6.63 to 8.75), and 25.44 ( $95 \%$ CI, 22.60 to 28.63) in Groups 2, 3, 4, 5, and 6, respectively. In Model 2, the respective odds ratios for metabolic syndrome compared with Group 1 were 2.92 (95\% CI, 2.30 to 3.69), 2.57 (95\% CI, 2.23 to 2.97 ), 5.16 (95\% CI, 4.44 to 6.00 ), 7.45 (95\% CI, 6.47 to 8.56), and 24.53 (95\% CI, 21.77 to 27.64 ) in Groups 2, 3, 4, 5, and 6 .

\section{DISCUSSION}

The following results were obtained through this study. 


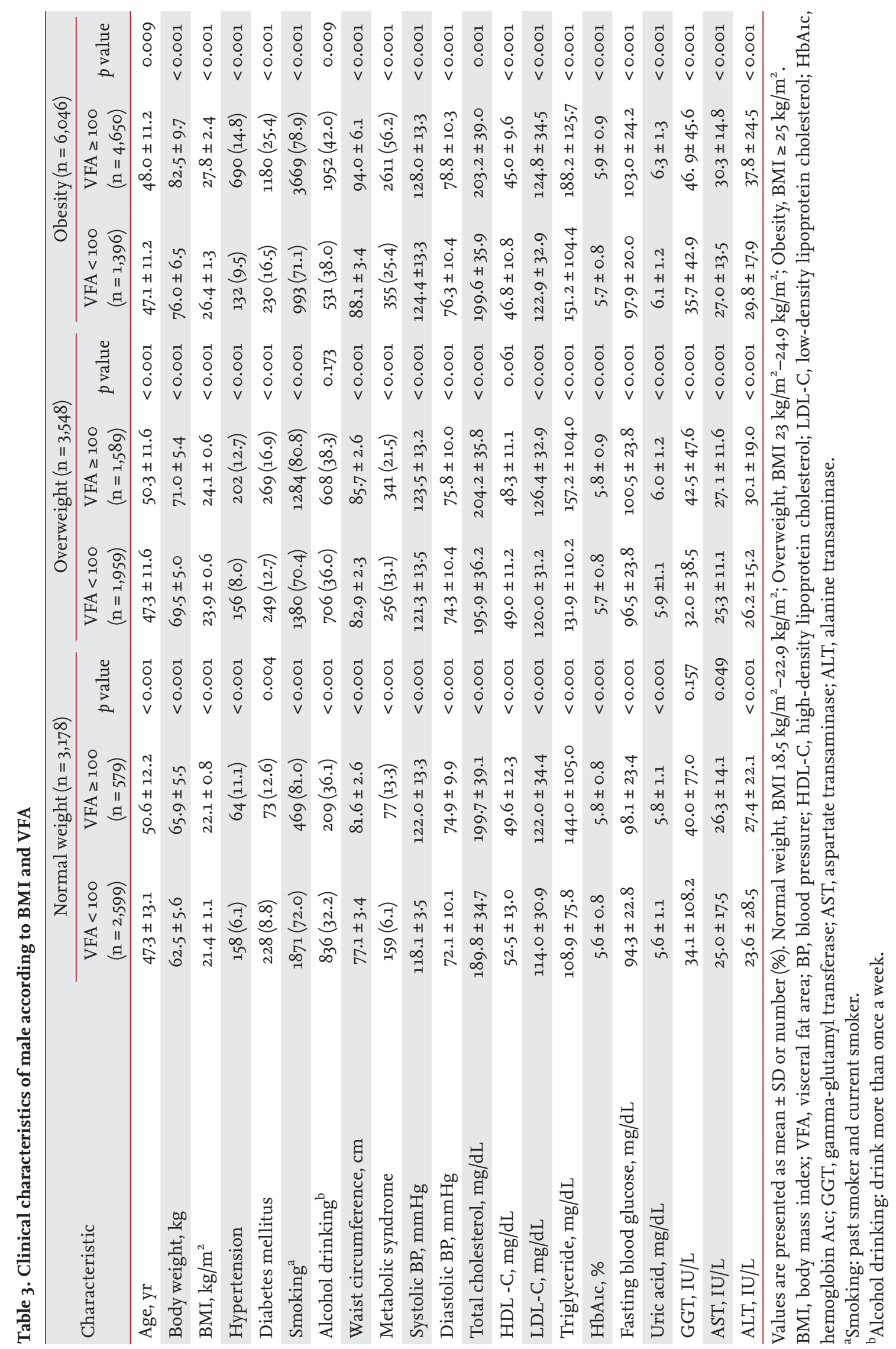




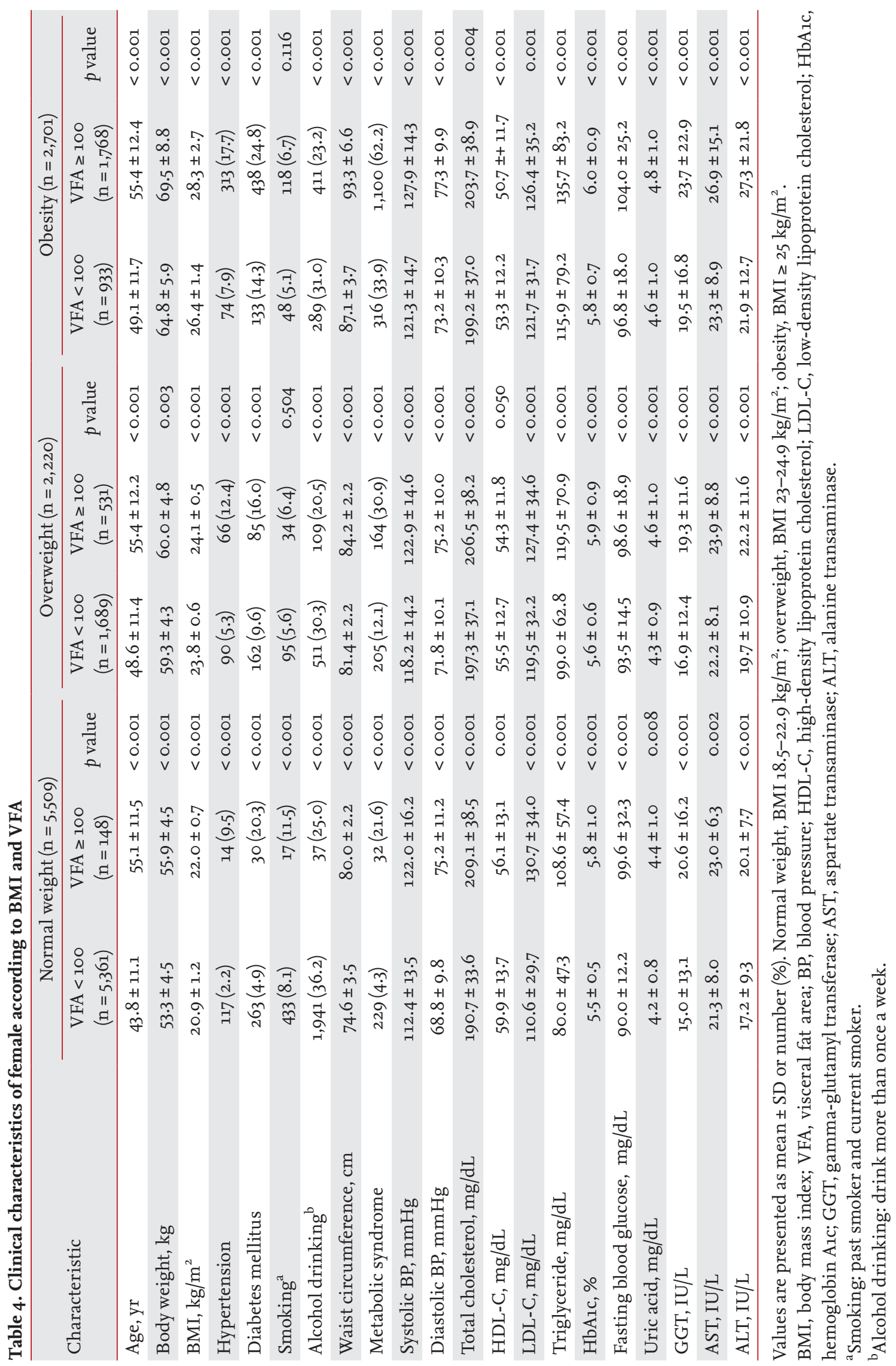


Table 5. The odds ratio of metabolic syndrome in the group stratified by the combination of body mass index and visceral fat area

\begin{tabular}{|c|c|c|c|c|c|c|c|}
\hline \multirow{2}{*}{ Variable } & \multirow{2}{*}{ No. } & \multirow{2}{*}{ Crude OR (95\% CI) } & \multirow{2}{*}{$p$ value } & \multicolumn{2}{|l|}{ Model 1} & \multicolumn{2}{|l|}{ Model 2} \\
\hline & & & & Adjusted OR (95\% CI) & $p$ value & Adjusted OR (95\% CI) & $p$ value \\
\hline Group 1 & 7,960 & 1.00 & & 1.00 & & 1.00 & \\
\hline Group 2 & 727 & $3.44(2.74-4.32)$ & $<0.001$ & $2.91(2.31-3.67)$ & $<0.001$ & $2.92(2.30-3.69)$ & $<0.001$ \\
\hline Group 3 & 3,648 & $2.82(2.45-3.25)$ & $<0.001$ & $2.64(2.29-3.04)$ & $<0.001$ & $2.57(2.23-2.97)$ & $<0.001$ \\
\hline Group 4 & 2,120 & $6.10(5.29-7.04)$ & $<0.001$ & $5.27(4.55-6.11)$ & $<0.001$ & $5.16(4.44-6.00)$ & $<0.001$ \\
\hline Group 5 & 2,329 & $7.90(6.89-9.05)$ & $<0.001$ & $7.62(6.63-8.75)$ & $<0.001$ & $7.45(6.47-8.56)$ & $<0.001$ \\
\hline Group 6 & 6,418 & $26.75(23.88-29.96)$ & $<0.001$ & $25.44(22.60-28.63)$ & $<0.001$ & $24.53(21.77-27.64)$ & $<0.001$ \\
\hline
\end{tabular}

Model 1. Adjust variable: age, sex; Model 2. Adjust variable: Model $1+$ diabetes mellitus, hypertension, alcohol, smoking. OR, odds ratio; CI, confidence interval.

First, VFA estimated by BIA correlated well with waist circumference and metabolic syndrome components. Although previous studies have shown similar results regarding VFA [11,12,16,17], our study demonstrated the usefulness of VFA estimation by BIA in a large number of participants. Visceral fat is known to be associated with insulin resistance and diabetes [18]. Our study revealed that VFA was also associated with $\mathrm{HbAic}$ level. Additionally, we found that VFA was associated with the level of uric acid. Uric acid is not included in the diagnostic criteria for metabolic syndrome, but recent studies have revealed that increased uric acid level plays a causative role in the pathogenesis of metabolic, renal, and cardiovascular diseases and leads to increased mortality [19-21].

Second, when participants were divided into six groups, the odds ratio for metabolic syndrome tended to increase with group number, and the odds ratio for metabolic syndrome in Group 6 was very high at 24.53 . Interestingly, when the normal-weight group was divided based on VFA, the odds ratio for metabolic syndrome in Group 2 (normal weight and VFA $\geq 100 \mathrm{~cm}^{2}$ ) was 2.92 compared with Group 1 (normal weight and VFA $<100$ $\mathrm{cm}^{2}$ ). Additionally, the odds ratio for metabolic syndrome in Group 2 was higher than that in Group 3 (overweight and VFA $<100 \mathrm{~cm}^{2}$ ). The odds ratio for metabolic syndrome increased dramatically from 7.45 to 24.53 when participants with obesity based on BMI were further divided based on VFA (Group 5 vs. Group 6). These results suggest that VFA is an important component and cause of metabolic syndrome. Previous studies have demonstrated that VFA is associated with metabolic syndrome components and have proposed $100 \mathrm{~cm}^{2}$ as a reasonable cut-off point for VFA, suggesting that VFA $<100 \mathrm{~cm}^{2}$ may be an important factor for improvement $[15,22]$. Visceral fat is associated with a greater cardiometabolic risk than subcutaneous fat or high BMI $[23,24]$. The adipocytokines induced by visceral fat are closely related with metabolic disorders [25], which develop into various metabolic and cardiovascular diseases. Visceral fat reduces the production of defensive adipocytokines such as adiponectin $[25,26]$. Changes in adiponectin in obesity play an important role in the development of metabolic and cardiovascular complications [25,27]. Circulating adiponectin level was found to correlate with VFA but not BMI in subjects with obesity [28].

The clinical significance of our study is as follows. The combination of BMI assessment and VFA determination by the BIA method allowed the diagnosis of individuals with an unrecognized but relatively high risk of metabolic syndrome (such as those with normal BMI and VFA greater than $100 \mathrm{~cm}^{2}$ ). In addition, our study confirmed that the risk of metabolic syndrome was significantly lower (OR, 7.45 vs. 24.53) when VFA was less than $100 \mathrm{~cm}^{2}$ in participants with obesity based on BMI. In other words, VFA (cut-off point of $100 \mathrm{~cm}^{2}$ ) may be a useful target for interventions to improve metabolic syndrome.

The present study had several limitations. First, our study was limited by its cross-sectional and single-center retrospective design. Second, exercise as a habitual parameter was not evaluated in the present study because the data was not correctly acquired. However, the influence of this limitation was reduced by the large sample size in our study. Third, VFA was not measured by a standard method such as CT, which is the most ac- 
curate and reliable method. However, VFA determined by BIA correlates well with that determined by CT $[17,29]$. In conclusion, this study demonstrated that the combination of BMI assessment and VFA determination by BIA may be a simple and useful method for predicting the risk of metabolic syndrome. The VFA by BIA may be a useful target for interventions to improve metabolic syndrome. Future interventional trials are needed to confirm the usefulness of targeting the VFA to improve metabolic syndrome.

\section{KEY MESSAGE}

1. Visceral fat area estimated by bioelectrical impedance analysis correlated well with waist circumference and metabolic syndrome components.

2. The combination of body mass index assessment and visceral fat area determination by the bioelectrical impedance analysis method allowed the diagnosis of individuals with an unrecognized but relatively high risk of metabolic syndrome.

3. Visceral fat area (cut-off point of $100 \mathrm{~cm}^{2}$ ) by bioelectrical impedance analysis may be a useful target for interventions to improve metabolic syndrome.

\section{Conflict of interest}

No potential conflict of interest relevant to this article was reported.

\section{Acknowledgments}

This study was supported by a grant from the National Health Insurance Service Ilsan Hospital, Republic of Korea. The authors acknowledge the effort of the health screening group at the National Health Insurance Service Ilsan Hospital, Republic of Korea.

\section{REFERENCES}

1. Finucane MM, Stevens GA, Cowan MJ, et al. National, regional, and global trends in body-mass index since 1980: systematic analysis of health examination surveys and epidemiological studies with 960 country-years and $9 \cdot 1$ million participants. Lancet 2011;377:557-567.

2. Bauer UE, Briss PA, Goodman RA, Bowman BA. Prevention of chronic disease in the 21st century: elimination of the leading preventable causes of premature death and disability in the USA. Lancet 2014;384:45-52.

3. Schienkiewitz A, Mensink GB, Scheidt-Nave C. Comorbidity of overweight and obesity in a nationally representative sample of German adults aged 18-79 years. BMC Public Health 2012;12:658.

4. Calle EE, Rodriguez C, Walker-Thurmond K, Thun MJ. Overweight, obesity, and mortality from cancer in a prospectively studied cohort of U.S. adults. N Engl J Med 2003;348:1625-1638.

5. Matsuzawa Y, Funahashi T, Nakamura T. The concept of metabolic syndrome: contribution of visceral fat accumulation and its molecular mechanism. J Atheroscler Thromb 2011;18:629-639.

6. Fox CS, Massaro JM, Hoffmann U, et al. Abdominal visceral and subcutaneous adipose tissue compartments: association with metabolic risk factors in the Framingham Heart Study. Circulation 2007;116:39-48.

7. Albu JB, Kovera AJ, Johnson JA. Fat distribution and health in obesity. Ann N Y Acad Sci 2000;904:491-501.

8. Gallagher D, Visser M, Sepulveda D, Pierson RN, Harris T, Heymsfield SB. How useful is body mass index for comparison of body fatness across age, sex, and ethnic groups? Am J Epidemiol 1996;143:228-239.

9. Carr DB, Utzschneider KM, Hull RL, et al. Intra-abdominal fat is a major determinant of the National Cholesterol Education Program Adult Treatment Panel III criteria for the metabolic syndrome. Diabetes 2004;53:2087-2094.

10. Dhaliwal SS, Welborn TA. Measurement error and ethnic comparisons of measures of abdominal obesity. Prev Med 2009;49:148-152.

11. Ranasinghe C, Gamage P, Katulanda P, Andraweera N, Thilakarathne S, Tharanga P. Relationship between body mass index (BMI) and body fat percentage, estimated by bioelectrical impedance, in a group of Sri Lankan adults: a cross sectional study. BMC Public Health 2013;13:797.

12. Unno M, Furusyo N, Mukae H, Koga T, Eiraku K, Hayashi J. The utility of visceral fat level by bioelectrical impedance analysis in the screening of metabolic syndrome: the results of the Kyushu and Okinawa Population Study (KOPS). J Atheroscler Thromb 2012;19:462-470.

13. Kushner RF, Gudivaka R, Schoeller DA. Clinical char- 
acteristics influencing bioelectrical impedance analysis measurements. Am J Clin Nutr 1996;64:423S-427S.

14. WHO Expert Consultation. Appropriate body-mass index for Asian populations and its implications for policy and intervention strategies. Lancet 2004;363:157-163.

15. Examination Committee of Criteria for 'Obesity Disease' in Japan; Japan Society for the Study of Obesity. New criteria for 'obesity disease' in Japan. Circ J 2002;66:987-992.

16. Lewin A, Pannier B, Meline J, Karusisi N, Thomas F, Chaix B. Residential neighborhood, geographic work environment, and work economic sector: associations with body fat measured by bioelectrical impedance in the RECORD Study. Ann Epidemiol 2014;24:180-186.

17. Sakamaki K, Maejima Y, Tokita Y, et al. Impact of the visceral fat area measured by dual impedance method on the diagnostic components of metabolic diseases in a middle-aged Japanese population. Intern Med 2016;55:1691-1696.

18. Koh H, Hayashi T, Sato KK, et al. Visceral adiposity, not abdominal subcutaneous fat area, is associated with high blood pressure in Japanese men: the Ohtori study. Hypertens Res 2011;34:565-572.

19. Kang DH, Park SK, Lee IK, Johnson RJ. Uric acid-induced C-reactive protein expression: implication on cell proliferation and nitric oxide production of human vascular cells. J Am Soc Nephrol 2005;16:3553-3562.

20. Nakagawa T, Mazzali M, Kang DH, et al. Hyperuricemia causes glomerular hypertrophy in the rat. Am J Nephrol 2003;23:2-7.

21. Hakoda M, Masunari N, Yamada M, et al. Serum uric acid concentration as a risk factor for cardiovascular mortal- ity: a longterm cohort study of atomic bomb survivors. J Rheumatol 2005;32:906-912.

22. Tatsumi Y, Nakao YM, Masuda I, et al. Risk for metabolic diseases in normal weight individuals with visceral fat accumulation: a cross-sectional study in Japan. BMJ Open 2017;7:e013831.

23. Despres JP, Moorjani S, Lupien PJ, Tremblay A, Nadeau A, Bouchard C. Regional distribution of body fat, plasma lipoproteins, and cardiovascular disease. Arteriosclerosis 1990;10:497-511.

24. Shah RV, Murthy VL, Abbasi SA, et al. Visceral adiposity and the risk of metabolic syndrome across body mass index: the MESA Study. JACC Cardiovasc Imaging 2014;7:1221-1235.

25. Kishida K, Funahashi T, Shimomura I. Adiponectin as a routine clinical biomarker. Best Pract Res Clin Endocrinol Metab 2014;28:119-130.

26. Weyer C, Funahashi T, Tanaka S, et al. Hypoadiponectinemia in obesity and type 2 diabetes: close association with insulin resistance and hyperinsulinemia. J Clin Endocrinol Metab 2001;86:1930-1935.

27. Santaniemi M, Kesaniemi YA, Ukkola O. Low plasma adiponectin concentration is an indicator of the metabolic syndrome. Eur J Endocrinol 2006;155:745-750.

28. Kishida K, Kim KK, Funahashi T, Matsuzawa Y, Kang HC, Shimomura I. Relationships between circulating adiponectin levels and fat distribution in obese subjects. J Atheroscler Thromb 2011;18:592-595.

29. Ogawa H, Fujitani K, Tsujinaka T, et al. InBody 720 as a new method of evaluating visceral obesity. Hepatogastroenterology 2011;58:42-44. 